\title{
¿CUÁNTO ESTAMOS DISPUESTOS A PAGAR POR EL SILENCIO?: UN ANÁLISIS CONTINGENTE PARA LA CIUDAD DE BARCELONA.
}

\author{
Carlos Marmolejo Duarte ${ }^{1}$ y Agustín Frizzera. \\ Departamento de Construcciones Arquitectónicas I Centro de Política de Suelo y Valoraciones. \\ Universidad Politécnica de Cataluña.
}

Remisión artículo: 20-04-2008

Palabras Clave: valoración contingente, valoración del ruido, Barcelona.

Resumen: El municipio de Barcelona posee un modelo de ciudad compacta caracterizado por una elevada densidad, en la que conviven diversas actividades económicas y residenciales, lo cual aunado a un diseño adecuado de los espacios públicos altamente utilizados debido al clima mediterráneo, produce niveles sónicos graves. Este problema tiene una especial incidencia no sólo en el uso del espacio público, sino también, en el nivel de bienestar experimentado dentro de las viviendas. La investigación que se reporta en este artículo intenta conocer el valor que, para el conjunto de la sociedad barcelonesa, tendría una eventual reducción del ruido urbano. A tales efectos se han realizado dos análisis contingentes que intentan conocer: 1) el nivel de importancia del ruido en relación a otros problemas y atributos urbanos y 2) la disposición a pagar por una eventual reducción del ruido. La importancia de la ausencia del ruido está en un $6^{\circ}$ lugar dentro de 11 características urbanas propuestas relacionadas con la accesibilidad, la jerarquía social y la calidad ambiental. De hecho el 50,7\% de los 405 respondedores/as declaró que el ruido tenía mucha o bastante importancia a la hora de elegir su localización residencial; mientras que el 50,8\% dijo que éste le provocaba bastante o mucha molestia. Sin embargo, cuando se les preguntó por el nivel de prioridad que tenía su reducción a través de políticas públicas éste quedó en $4^{\circ}$ lugar dentro de un conjunto de 5 acciones propuestas. De media las personas estarían dispuestas a pagar por la reducción sónica ofrecida 3,39 euros por mes y persona, es decir, aproximadamente el 0,28\% de la renta familiar disponible. El análisis econométrico de las respuestas, con ayuda de un SIG, sugiere que detrás de la DAP está el nivel de perjuicio producido por el ruido, el nivel de renta, la percepción de otros factores ambientales, y el nivel de aislamiento de sus viviendas contra el ruido. Estos resultados son coherentes con la teoría y muy similares a los de otras investigaciones de valoración contingente.

\footnotetext{
${ }^{1}$ Autor de contacto: carlos.marmolejo@upc.edu
} 


\section{Introducción}

Nuestras vidas están repletas de sonidos, los sonidos nos elevan, nos enaltecen; sin embargo, cuando un sonido resulta excesivo y molesto para quien lo oye se convierte en un "ruido" (del latín rugitus, rugido). El problema de la valoración del ruido en los ambientes urbanos es complejo por dos razones: en primer lugar porque el valor es una construcción social ampliamente influida por la percepción, y en segunda porque el silencio es un bien público, y por tanto no tiene precios explícitos. La percepción a su vez en si misma compleja porque está influida por aspectos físicos, culturales y subjetivos propios de la persona que percibe el sonido (Daumal, 2001).

Dentro de las herramientas de la valoración ambiental, dos han sido las aproximaciones a la valoración del ruido: de la familia de las preferencias reveladas, el método de precios hedónicos (en lo sucesivo $\mathrm{PH}$ ); y de la familia de las preferencias declaradas, la valoración contingente (VC). Mientras que el método de $\mathrm{PH}$ intenta inferir, por medio de técnicas de análisis multivariante, el precio implícito de las variables ambientales, la VC extrae directamente la disposición a pagar (en lo sucesivo DAP) por ellas, en tanto se entiende modifican el nivel de bienestar de los individuos. En definitiva mientras que el primer método utiliza el mercado de los bienes privados como mecanismo de valoración de los públicos, en el segundo se realiza una emulación de un hipotético mercado de provisión de bienes ambientales (Riera, 1994). A pesar de que ambos métodos intentan llegar a un mismo fin, existen diferencias sustanciales tanto en su aplicabilidad como en el valor que son capaces de capturar. Así, mientras el método de PH requiere de la existencia de un mercado de bienes privados, por ejemplo el inmobiliario, sobre el cual se reflejen las externalidades de los bienes públicos, en el segundo método este supuesto no es necesario. Más en el fondo, mientras que el método de VC es fácil que se aprehendan valores de no uso, como los de oportunidad, existencia y legado, en el método de los $\mathrm{PH}$ básicamente se internalizan valores de uso. Esto abre una brecha importante sobre los ámbitos en los que se pueden utilizar estas metodologías y sobre los resultados que ambas arrojan, véase a Brookshire et al. (1982).

Cabe señalar que en la economía ambiental la DAP no es más que una vía para conocer en qué medida las personas valoran el incremento en el nivel de bienestar que origina un incremento en el nivel de un bien público. En ningún momento el método asume que efectivamente las personas tengan que pagar por beneficiarse del bien propuesto cuando se valora la desaparición de externalidades negativas emitidas por otros. Quizás, en este caso, sería más coherente utilizar la vía de la disposición a ser compensado (DAC), siendo el que paga el emisor de las externalidades, y el que recibe quien tiene derecho a disfrutar de unas condiciones ambientales adecuadas. Sin embargo, esta última aproximación, a pesar de que tiene el mismo significado teórico que la DAP, es decir encontrar la variación compensatoria suele arrojar sesgos más difíciles de controlar, y por tanto su utilización es escasa. La variación compensatoria es la cantidad monetaria que pagaríamos por disfrutar de un incremento en la calidad ambiental manteniendo nuestro nivel de utilidad, y que además, haría indiferente la alternativa de no pagar nada, mantener el estado de la calidad ambiental y mantener nuestro nivel de utilidad (Riera, 2005). En definitiva esta variación mide, en unidades monterías, lo que se gana o se pierde con un cambio en el nivel de bienestar. 
En este artículo se reportan los resultados de una investigación basada en una VC que intenta conocer, para la ciudadanía del municipio de Barcelona: 1) la importancia relativa que el ruido tiene en la elección de la localización residencial y 2) la DAP por una eventual reducción del nivel de ruido. Este trabajo ha sido desarrollado en el contexto del Curso de Valoración Ambiental de la UPC ${ }^{2}$. El resto del artículo se estructura así: primero se hace una pequeña revisión de las dos técnicas utilizadas en la literatura de la valoración del ruido, luego se explica la estructura de la encuesta utilizada, enseguida se exponen los resultados del análisis de ranquin contingente y finalmente se hace lo propio con el análisis de la DAP. El artículo concluye sintetizando el trabajo realizado y los principales hallazgos encontrados.

\section{La valoración hedónica y contingente del silencio.}

El método de $\mathrm{PH}$ asume que el precio de mercado de los activos inmobiliarios internaliza los precios implícitos de las externalidades ambientales. De manera que las localizaciones mejor provistas de servicios ambientales, manteniendo igual todo el resto de factores, tienen un mayor precio, en tanto proveen de una utilidad mayor a sus usuarios. Por tanto, el valor del suelo no es más que la integración del valor marginal de los atributos locacionales como la accesibilidad, la jerarquía social y la calidad del medio ambiente (natural o antropeizado). Precisamente por este carácter multivariante, el método de los precios hedónicos parte de la utilización de la estadística para inferir el valor de cada uno de estos factores, máxime cuando en la práctica lo que se utiliza no es el precio del suelo, sino el de los edificios acabados, y en este sentido es necesario controlar otros factores como la tipología, el estado de conservación, la calidad de los acabados, etc.

La metodología asume que los hogares, al adquirir o alquilar una vivienda, son plenamente conscientes no sólo del nivel de ruido al que estarán expuestos sino sobre todo del nivel de merma que éste provocará sobre su nivel de bienestar. Esto es mucho asumir, porque las asimetrías informativas en el mercado inmobiliario, en tanto los bienes no son perfectamente sustituibles entre sí, son enormes, y porque la percepción es compleja en tanto es difícil evaluar el impacto que producirá un evento que aún no se ha experimentado. Estas situaciones pueden provocar inestabilidad en la DAP implícita (Becker y Lavee, 2003). En teoría si los hogares viesen insatisfechas sus expectativas tendrían que vender inmediatamente el activo y buscar otro, reajustando de esta manera el precio, lo cual no ocurre exactamente así en la realidad, debido a los significativos costes de transacción (mudanzas, impuestos, comisiones, servicios jurídicos, gastos registrales, etc.). Adicionalmente en países en donde el cambio residencial a lo largo del tiempo es escaso, como históricamente lo ha sido en España, el ajuste del precio por esta vía es dudoso. Por otra parte el método asume que los hogares, en aras de maximizar su función de utilidad, eligen la localización residencial donde el nivel de cada atributo es tal que su disposición marginal a pagar para cada uno se equipara a su precio implícito (Rosen, 1974). Lo cual, de hecho, es difícil que ocurra debido a

\footnotetext{
${ }^{2}$ Este curso forma parte de los actuales estudios de Máster Oficial de Investigación en Gestión y Valoración Urbana. Esta investigación se implementó con la ayuda del alumnado de la edición 2006 de dicho curso el cual participó en la definición de la encuesta y su aplicación: Carlos Roca, César Sánchez, Claudia Pérez, Eileen Tuñón, Irune Sacristán, Jimena de Gortari, Natalia Romero, Núria Romera, Mariela Offermann y Paulina Monreal. Asimismo, se contó con la asesoría de los profesores doctores Francesc Daumal y Josep Roca, especialistas en acústica y valoraciones respectivamente. La georeferenciación de las encuestas en el sistema de información geográfica corrió a cargo de Luis Miguel Llamas. A todas estas personas los autores agradecen sus aportaciones y eximen de cualquier error en el diseño, implementación, análisis e interpretación de los resultados.
} 
que los hogares difícilmente pueden evaluar simultáneamente y con suficiente profundidad todos los atributos que componen la propiedad, y además tener a su disposición una oferta amplia donde elegir precisamente aquel inmueble cuyos precios implícitos se equiparan a las disposiciones marginales de pago. A pesar de estas fronteras teóricas y prácticas el método de los $\mathrm{PH}$ ha sido el más utilizado en la valoración marginal del silencio tanto en ambientes urbanos como rurales.

Para medir el impacto del ruido sobre el valor de los inmuebles Walters (1975) desarrolló el Noise Depreciation Sensibility Index (NDSI). EI NDSI representa la reducción porcentual en el precio de los inmuebles por cada unidad de variación del sonido. Habitualmente esta unidad de variación es un indicador simple de la intensidad sónica medida en dBA (con patrones como el Leq, Ldn o el L10), y excepcionalmente es un indicador compuesto como el NEF, ANEF o el $\mathrm{NNI}^{3}$.

A efectos de estimación directa del NDSI en la literatura los modelos utilizados son del tipo (1).

$$
\operatorname{LnP}=C+\sum_{i}^{n} B_{i} X_{i}
$$

En (1) $L n P$ es el logaritmo neperiano del precio de mercado de los bienes privados (usualmente el precio de las viviendas), $C$ es la constante del modelo, y $B$ es el coeficiente de cada una de las $i$ covariables o factores $X$ dentro de los que se incluyen los indicadores de la calidad ambiental, como la intensidad del sonido. En otras palabras (1) mide la elasticidad del precio de mercado de los bienes privados ante la variación de las variables independientes. Diferentes estudios empíricos han puesto de relieve la variación del NDSI en diferentes geografías en Marmolejo (2008) se ha realizado una revisión de ellos para el caso del ruido vehicular (principal causante del ruido urbano ambiental). En síntesis los estudios revisados por él han encontrado que el NDSI adopta un valor medio de $0,8 \%$ con un recorrido de $0,08 \%$ a $2,36 \%$. Es decir de media el valor de los inmuebles analizados se reduce en un $0,8 \%$ por cada unidad (p.e.: $d B A L_{e q}$ ) que incrementa la intensidad del sonido. Es interesante ver como algunas investigaciones reportadas en dicho estudio como el de Becker y Lavee han detectado que el impacto del ruido vehicular es más alto en los entornos suburbanos en comparación con los entornos urbanos; lo cual sugiere una mayor apreciación del silencio en aquellas áreas que se espera sean, por antonomasia, silenciosas. Para el caso del ruido aeroportuario el NDSI adopta valores más severos. En una revisión de estudios de PH Marmolejo y Romano (2008) han reportado que el NDSI en entornos residenciales afectados por instalaciones aeroportuarias varía entre el $0 \%$ y el $10 \%$ con una media de $3 \%$, un recorrido y una media más amplia, y que resulta coherente con el efecto esperado que tiene este ruido tan específico y de un impacto más localizado.

A diferencia del método de PH la VC ha tenido una menor aplicación empírica en la estimación del valor marginal del silencio. Este hecho puede ser explicado por el mayor coste para aplicar la encuesta y la mayor dificultad para evitar los sesgos estratégicos que puede conllevar el uso de esta metodología, y por tanto el escepticismo de algunos investigadores para utilizarla. Sin embargo, el informe Blueribbon de la National Oceanic and Atmospheric Administration de

\footnotetext{
${ }^{3}$ Estos indicadores combinan el tono, la intensidad, el número de eventos (p.e.: aterrizajes o despegues en el caso del ruido aeroportuario)
} 
EEUU de 1993 ha avalado el uso de la VC en la valoración de las externalidades ambientales, y en general, de los bienes públicos.

Vainio (2001) ha aplicado el método de VC en Helsinki, mediante 418 encuestas válidas, encontrando que la DAP media es de 22 euros (de dicho año) por dB de reducción (por encima de 55 dBA) por hogar y por año. Navrud (2000) ha encontrado, en un estudio aplicado en Oslo que la DAP por beneficiarse de una reducción del ruido viario por hogar y por año variaba entre 165 y 275 euros, asumiendo que dicha reducción ofrecida equivalía a $10 \mathrm{dBA}$. En su encuesta las personas entrevistadas estaban expuestas a ruidos de $65 \mathrm{~dB}$ y más; sin embargo, el autor no encontró una correlación significativa entre el nivel de perturbación sónica y la DAP. Al contrario Bjorner (2004) en un análisis aplicado en Copenhague ha encontrado una correlación clara entre el nivel de perturbación declarado por los respondedores/as y su DAP. Asimismo este autor encontró que las personas con altos niveles de educación y con niños viviendo en el hogar estaban dispuestas a pagar más por la reducción sónica ofrecida, manteniendo igual el resto de factores. Estos hallazgos son coherentes con la teoría porque es previsible que las personas con mayores niveles educativos empleen el tiempo doméstico en actividades como la lectura, la realización de trabajos en casa, es decir actividades fácilmente perturbables por el ruido, de la misma manera que lo es el sueño de los infantes.

En España, Barreiro et al. (2005) han realizado un estudio de VC en Pamplona mediante el uso de 600 encuestas. Su aproximación novedosa utiliza el one and one-half bounded method desarrollado y aplicado por Cooper and Hanemann en 1995 y Cooper et al. en el año 2001 para encontrar el valor marginal a pagar por una reducción sónica. La principal ventaja de esta innovación es la minimización de los sesgos estratégicos. Los resultados muestran que la DAP está entre los 26 y 29 euros por hogar y por año para una reducción sónica equivalente a pasar de la intensidad de un día laboral a una hora laboral a otra equivalente a un día laboral a las 21:30; o de pasar de un día laboral a una hora laboral a otra equivalente a un domingo por la mañana. Los autores argumentan que, más allá del nivel de perjuicio provocado por el ruido, hay factores socioeconómicos que influencian la DAP. En su estudio el hecho que los respondedore/as tuviesen estudios universitarios afecta positiva y significativamente la DAP, así como la credibilidad del vehículo de provisión ofertado. ${ }^{4}$ En términos de proporción con la renta la DAP representa un $0,27 \%$ del ingreso medio anual familiar, que es un tanto menor que el $0,32 \%$ reportado por el trabajo de Vainio (2001). Sin embargo, al igual que en el caso de los resultados del método de los $\mathrm{PH}$, se ha de tener en cuenta que detrás de la DAP hay factores como la cultura, el nivel de exposición a los espacios exteriores -fuertemente influido por la climatología- y otros aspectos edificatorios que pueden hacer variar esta valoración.

En Barcelona Daumal y de Gortari (2006) han realizado un estudio sobre la relación que existe entre el ruido y el precio de la vivienda. Sobre la base de 207 encuestas de tipo sociológico aplicadas en el Eixample, uno de los distritos centrales más diversos y densos de la ciudad, han encontrado que un $66 \%$ de los respondedores/as consideran molesto el ruido de la calle. Asimismo, reportan que los residentes cuyas viviendas dan hacia el interior de las manzanas valoran más el silencio, en tanto están menos expuestos al ruido, y además se consideran privilegiados frente a las personas cuyas viviendas tienen frente a las vías públicas. Sin embargo, los respondedores/as a pesar de reconocer alguna existencia entre el valor de la

\footnotetext{
${ }^{4}$ La encuesta incluía una pregunta que evaluaba cuan eficaz crean los respondedores eran las políticas de lucha contra el ruido ofrecidas como vehículo de provisión.
} 
vivienda y el nivel de ruido; dijeron que el valor del suelo estaba más influido por otros factores como las comunicaciones o la oferta de servicios.

Más allá de la versión más habitual de la VC otros autores como Saelensminde (1999) o Scarpa y Willis. (2006) han usado modelos de elección discreta para extraer la DAP por la reducción del ruido, a efectos de minimizar los sesgos estratégicos.

\section{Magnitud del problema sónico en Barcelona y diseño de la valoración.}

Barcelona (1,6 millones de personas, $100 \mathrm{Km}^{2}$ de los cuales 64 urbanizados) es el corazón de la segunda área metropolitana de la península Ibérica. Como ciudad mediterránea y compacta que es, sufre de problemas de ruido considerables, en tanto el denso tejido urbano está caracterizado por una gran diversidad de usos que conviven espacialmente en un esquema de actividades mixtas. Además, el buen clima de la región y el diseño de los espacios públicos, favorece la realización de actividades al aire libre, dentro de las cuales, las ejercidas por el turismo han ganado de manera progresiva protagonismo. Todo esto, aunado a una relativamente escasa presencia de áreas verdes favorece la generación y propagación del sonido.

\subsection{El problema del ruido urbano en Barcelona.}

El penúltimo mapa sónico realizado en 1997 ayuda a ilustrar la magnitud del problema. En dicho ejercicio se realizaron 95 mediciones de 30 minutos y 4.715 mediciones de 10 minutos durante días laborables sin vientos ni lluvias obteniendo los resultados de la tabla 1. En dicha tabla se observa cómo la mayor parte de las mediciones diurnas se encuentran según los autores del estudio en un nivel "tolerable" (de 65 a 75 dB diurnos), mientras que un 37,2\% de las mediciones nocturnas se han de mejorar. En cambio, si se tomasen como referencia los niveles recomendados por otras organizaciones como la de la OCDE la cual considera que a partir de 55-60 dBA el ruido causa molestia, entre 60-65 dBA la molestia es considerable, y por encima de los 65 dBA surgen perturbaciones en el comportamiento y síntomas de daño grave (García y Garrido, 2003, pág. 98), tendríamos que en horario diurno el 76,6\% de los puntos podrían comprometer gravemente el comportamiento y la salud, mientras que en horario nocturno un $91,5 \%$. Este es uno de los costes sociales de vivir en una ciudad compacta, diversa y densa. Ante este panorama la administración local ha realizado una sería de políticas públicas como: campañas de concienciación ciudadana, vigilancia sónica, peatonalización, inversión de prioridad de tránsito y repavimentación de los principales viales, en tanto, es el tráfico viario, según han demostrado los estudios, el principal causante de este mal. ${ }^{5}$

\footnotetext{
${ }^{5}$ Si se comparan los resultados del mapa acústico de 1990 con el de 1997 se ve el resultado de alguna de estas políticas municipales y de la propia mejora de las emisiones sónicas del transporte, en concreto la intensidad sónica ha disminuido en 7 distritos entre $-2,0 \mathrm{~dB}$ y $-0,2 \mathrm{~dB}$ y sólo en 3 distritos ha incrementado en menos de $+0,05 \mathrm{~dB}$.
} 
Tabla 1 Intensidad sónica en Barcelona en 1997 según 95 mediciones del mapa sónico municipal

\begin{tabular}{ccccr}
\hline $\begin{array}{c}\text { Intensidad } \\
\text { diurna }\end{array}$ & $\begin{array}{c}\text { Intensidad } \\
\text { nocturna }\end{array}$ & $\begin{array}{c}\text { Tolerancia } \\
\text { según los } \\
\text { autores del } \\
\text { estudio }\end{array}$ & $\begin{array}{c}\text { \% puntos } \\
\text { medidos en } \\
\text { horario diurno }\end{array}$ & $\begin{array}{c}\text { \% puntos } \\
\text { medidos en } \\
\text { horario } \\
\text { nocturno }\end{array}$ \\
\hline$<65 \mathrm{~dB}$ & $<55 \mathrm{~dB}$ & Buena & $23,4 \%$ & $8,5 \%$ \\
\hline $65 \mathrm{a} \mathrm{75 \textrm {dB }}$ & $55 \mathrm{a} 65 \mathrm{~dB}$ & Tolerable & $63,8 \%$ & $54,3 \%$ \\
\hline$>75 \mathrm{~dB}$ & $>65 \mathrm{~dB}$ & a mejorar & $12,8 \%$ & $37,2 \%$ \\
\hline \multicolumn{5}{c}{$100,0 \%$} \\
\hline Fuente: elaboración propia sobre la base de los resultados del Mapa Acústico 1997 (Ajuntament de Barceloni
\end{tabular}

\subsection{Estructura de la encuesta contingente}

La estructura de la encuesta realizada en esta investigación está formada por tres partes: 1) contextualización, 2) ranquin contingente, valoración contingente DAP, y 3) Información socioeconómica y de control. La parte de contextualización tiene el objetivo de hacer recordar a las personas encuestadas la experiencia cotidiana que tienen con el ruido ambiental. En este sentido se les pidió que mencionaran, dentro de una escala ordinal ad hoc, el nivel de molestia que les provoca el ruido que proviene del exterior de su vivienda; además se les preguntó a qué hora del día, durante los días de la semana y los fines de semana, dicha externalidad les provocaba una mayor perturbación. Asimismo, se les pidió que especificaran la fuente emisora. De acuerdo con Barreiro et al. (2005) esta primera fase de contextualización prepara a los encuestados para la valoración contingente propiamente dicha, en tanto, les permite recordar la perturbación que éstos experimentan cuando el ruido exterior irrumpe sus actividades domésticas.

La valoración contingente es la segunda parte de la encuesta. La reducción de ruido ofrecida equivale a pasar del "nivel actual de ruido en un día laboral durante una hora punta a otro nivel inferior como el que se experimenta en un día laboral a las 21:00 hrs". Este ofrecimiento permite evaluar la reducción en función de la propia experiencia de las personas, a costa de no poder concretar una cantidad precisa del ofrecimiento medida en $\mathrm{dB}$. En todo caso el ofrecimiento por una reducción específica de la intensidad en dB sería artificioso, en tanto las personas encuestadas no son expertas en la mensuración de esta faceta del sonido. Nótese que una eventual reducción del ruido que se suscita en una hora punta a otro en un horario nocturno comporta no sólo la reducción de la intensidad, sino también implica un cambio de las fuentes emisoras, de las frecuencias, de la intermitencia de emisión, y también de las actividades que son perturbadas en la actividad cotidiana de los encuestados.

Para reducir la tasa de rechazo, se pensó en que el vehículo de provisión partiese de una directriz europea y no fuese, en origen, una iniciativa local. En concreto los encuestados fueron informados de la existencia de un programa de reducción del ruido en los principales centros urbanos formado por una repavimentación con materiales de baja sonoridad, la 
instalación de paneles sono-absorbentes, el incremento de las barreras verdes, el soterramiento de algunas vías de alta velocidad y la optimización sónica del tránsito vehicular. Dicho programa, sería implementado por los ayuntamientos y financiado a través de una tasa mensual por persona. La razón para elegir este vehículo de pago es sencilla y responde al hecho que en España la ciudadanía está bastante habituada a realizar contribuciones de esta naturaleza destinadas a financiar servicios específicos, en este caso la reducción del ruido ambiental.

Una vez expuesto el escenario de provisión y explicado el vehículo de pago, se procedió a extraer la DAP. La escala de la disposición de pago es continua basada en una aproximación de subasta: primero se preguntó si se estaría dispuesto a pagar más o menos que un valor de salida, en seguida se volvió a preguntar si se estaba dispuesto a pagar más o menos que un segundo valor de referencia, finalmente se pidió que se concretase de manera abierta la cantidad. Tanto el valor de salida, como el de segunda referencia fue producto de la calibración previa de la encuesta. En el caso de negativa de pago, y con el objeto de identificar las respuestas de protesta, se pidió que se motivase la indisposición a pagar.

Alternativamente a la valoración contingente DAP se realizó una valoración por el método de ranquin contingente. Primero las personas encuestadas valoraron ordinalmente el grado de importancia que le darían al nivel del ruido en el evento que pudiesen elegir una localización para vivir. Luego se pidió que se valorase ordinalmente la importancia que tienen diferentes atributos espaciales en la elección residencial, tales como: la proximidad al transporte ferroviario, las buenas comunicaciones viales, la presencia de parques y equipamientos, el estado y la calidad de la urbanización, la disponibilidad de comercios de conveniencia, la oferta de bares, restaurantes y terrazas de ocio, la seguridad pública, el prestigio social de la zona, la ausencia de ruido y la ausencia de actividades industriales molestas. Para conocer la importancia relativa del ruido como problema ciudadano, frente a otros problemas, se realizó una segunda aproximación. En concreto se pidió a los encuestados que fijasen el nivel de prioridad con el cual el Ayuntamiento tendría que atender problemas relacionados con: la mejora de la política de vivienda, la mejora urbanística de los barrios con la dotación espacios verdes, la construcción de equipamientos, la mejora del transporte público y la reducción del ruido.

La tercera parte de la encuesta tiene por objeto recoger aquellos aspectos socioeconómicos y de la vivienda de las personas que podrían explicar la valoración del ruido. En lo relativo a la vivienda se recogieron los factores que pueden potenciar la exposición de las personas al ruido exterior cuando éstas están en su vivienda. En concreto se recogió el tipo de carpintería y vidrios, las posibles obras de aislamiento acústico ${ }^{6}$, el nivel real de la vivienda en relación a la vía pública, el hecho que las viviendas tuviesen frente a la vía, al patio de manzana o ambos, lo mismo en relación al dormitorio de la persona encuestada y el tipo de vía pública en el que está su vivienda (p.e.: avenida, calle, vía rápida, etc.), asimismo se pidió se declarase el número de años que el o la respondedora llevaba viviendo en su domicilio actual.

\footnotetext{
${ }^{6}$ Nótese que el nivel de aislamiento tiene dos significados, por una parte, se espera que las personas más sensibles al ruido hagan obras de aislamiento siempre y cuando éstas estén dentro de sus posibilidades, y por otra, las personas que residen en viviendas aisladas es probable estén menos afectadas por el ruido anterior.
} 
Desde la perspectiva socioeconómica se recogió la edad, el tipo de hogar y en su caso de familia, el número de hijos, el número de hijos menores de 15 años, la relación con la actividad, en su caso la escala socioprofesional, el nivel de estudios y el sexo. Factores todos ellos que potencialmente podrían tener alguna relación con la perturbación percibida por el ruido. La encuesta fue aplicada ${ }^{7}$ en la primavera del 2006 con 405 unidades válidas que fueron respondidas por personas mayores de 18 años.

\section{Resultados del ranquin contingente.}

En general el ruido es considerado como una molestia por el $64 \%$ de los respondedores, la mayor parte considera que el ruido les perturba bastante. Asimismo, casi la mitad (50,6\%) de los/as respondedores dijeron que el nivel sónico de la zona sería importante ante una eventual elección residencial como se detalla en la tabla 2. De hecho la tabla 3 documenta, a través del análisis de la chi-cuadrada de Pearson y del rho de Spearman que el nivel de molestia del ruido está correlacionado positivamente con cuán importante es para las personas el ruido a la hora de elegir el sitio donde vivir, esto soporta la idea que las respondedoras/es fueron coherentes en sus respuestas a lo largo de la encuesta.

Tabla 2 Nivel de molestia del ruido e importancia de éste en la elección residencial.

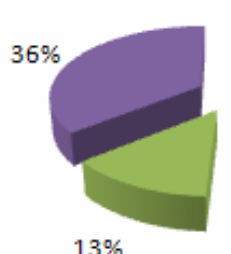

$13 \%$

Nivel molestía ruido

(f)

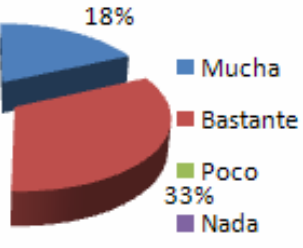

Nada

\begin{tabular}{lrc} 
& (f) & $(\%)$ \\
\hline & & \\
\hline Mucha & 73 & $18,2 \%$ \\
\hline Bastante & 131 & $32,6 \%$ \\
\hline Poco & 54 & $13,4 \%$ \\
\hline Nada & 144 & $35,8 \%$ \\
\hline & & \\
\hline Total general & 402 & $100,0 \%$ \\
\hline
\end{tabular}

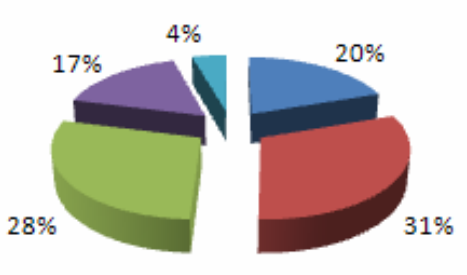

Mucha $\quad$ Bastante Normal

alguna Ninguna

Importancia del ruido en la elección de localización de la vivienda

\begin{tabular}{|c|c|c|}
\hline & (f) & $(\%)$ \\
\hline Mucha & 80 & $19,8 \%$ \\
\hline Bastante & 125 & $30,9 \%$ \\
\hline Normal & 115 & $28,4 \%$ \\
\hline Alguna & 67 & $16,5 \%$ \\
\hline Ninguna & 18 & $4,4 \%$ \\
\hline Total general & 405 & $100,0 \%$ \\
\hline
\end{tabular}

Fuente: elaboración propia

\footnotetext{
${ }^{7} 113$ encuestas fueron telefónicas, mientras que 292 presenciales.
} 


\section{Tabla 3 Relación entre el nivel de molestia del ruido y la importancia de éste en la elección residencial.}

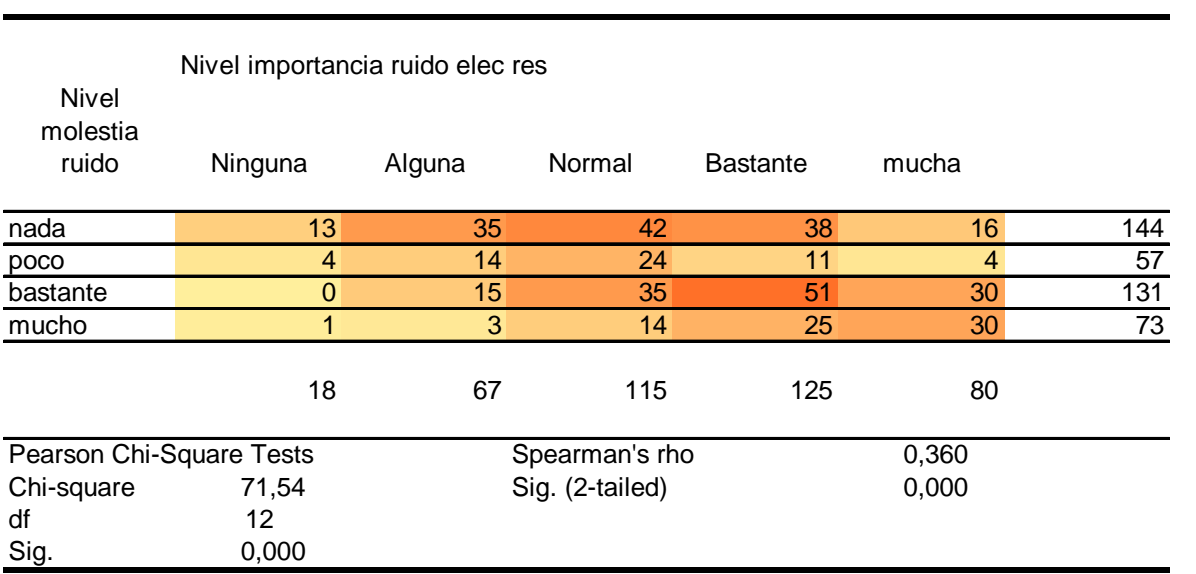

The Chi-square statistic is significant at the 0.05 level.

Correlation is significant at the 0.01 level (2-tailed).

Fuente: elaboración propia

La tabla 4 resume el resultado de la valoración relativizada de la ausencia del ruido frente a otros atributos espaciales a la hora de elegir la localización residencial. En general los respondedores/as dijeron que la seguridad pública ${ }^{8}$ es el primer factor decisivo, seguido de la ausencia de industrias molestas, de la accesibilidad tanto en transporte público como privado, y de la dotación de equipamientos. En sexto lugar quedó el ruido, aunque indirectamente este está representado por la ausencia de industrias que, entre otras externalidades negativas, generan ruido. Ahora bien, cuando la muestra se segmenta en función de la molestia del ruido se observa que las personas que dicen que el ruido les molesta priman más la ausencia de ruidos (posición 5 del ranquin) por sobre la buena comunicación vial (que para este grupo queda en la sexta posición). Esto podría indicar que las personas más afectadas por el ruido viven cerca de las vías de comunicación principales y por esta razón tienen una mayor perturbación acústica, que las hace valorar más el silencio en vez de las comunicaciones viales. El test ANOVA compara la similitud estadística de las medias, y para este caso demuestra que solamente en la valoración del ruido y de los jardines-equipamientos, las respuestas son significativamente diferentes entre las personas perturbadas y las no perturbadas por el ruido. El hecho que las personas perturbadas por el ruido valoren más la presencia de jardines y equipamientos en comparación con las no perturbadas refuerza la hipótesis anteriormente expuesta, por cuanto estos sitios suelen generar menos ruido que las vías de comunicación.

\footnotetext{
${ }^{8}$ Este atributo podría estar sobrevalorado porque justo en la época de encuestamiento salió a la luz un conjunto de asaltos y robos a viviendas unifamiliares, seguramente magnificado por los medios informativos.
} 


\section{Tabla 4 Valor relativo del silencio frente a otros atributos locacionales $(0=\sin$ importancia, 4= muy importante)}

\begin{tabular}{|c|c|c|c|c|c|}
\hline & \multicolumn{3}{|c|}{ Le molesta el ruido } & \multicolumn{2}{|c|}{ Test ANOVA } \\
\hline & $\begin{array}{l}\text { Ranquin } \\
\text { general }\end{array}$ & No & $\mathrm{SI}$ & $\mathrm{F}$ & Sig. \\
\hline Proximidad a estación metro o tren & 3 & 3,2 & 3,2 & 0,00 & 0,98 \\
\hline Buena comunicación vial & 4 & 3,2 & 3,1 & 0,57 & 0,45 \\
\hline Presencia de jardines y equipamientos & 5 & 3,0 & 3,2 & 5,94 & 0,02 \\
\hline Buena calidad y conservación de la urbanización & 7 & 3,0 & 3,1 & 0,80 & 0,37 \\
\hline Disponibilidad de comercios de conveniencia & 9 & 2,9 & 2,9 & 0,15 & 0,70 \\
\hline Disponibilidd de bares, restaurantes y terrazas & 11 & 2,0 & 1,9 & 0,83 & 0,36 \\
\hline Seguridad pública & 1 & 3,4 & 3,5 & 1,79 & 0,18 \\
\hline Prestigio social de la zona & 10 & 2,3 & 2,2 & 0,57 & 0,45 \\
\hline Ausencia de ruidos molestos & 6 & 2,9 & 3,2 & 5,44 & 0,02 \\
\hline Proximidad a los servicios sanitarios & 8 & 2,9 & 3,0 & 0,03 & 0,86 \\
\hline Ausencia de act. industriales molestas & 2 & 3,3 & 3,4 & 1,49 & 0,22 \\
\hline
\end{tabular}

*Estadísticamente significativo al 95\% de confianza

La segunda aproximación es la prioridad de actuación del Ayuntamiento, como se había dicho antes, los/as respondedores/as declararon cual, según ellos, era el orden de apremio en la solución de problemas en la agenda municipal. La tabla 5 inferior detalla que el acceso a la vivienda es la prioridad principal, seguida de la mejora en el transporte público, y el mejoramiento de las áreas verdes. Por su parte la reducción del ruido quedó en cuarto lugar sólo antes de la mejora de los equipamientos. A diferencia del análisis anterior en este caso, el análisis ANOVA no revela diferencias estadísticamente significativas en la forma en cómo respondieron las personas separándolas en dos grupos según la molestia. Ambas aproximaciones sugieren que si bien a efectos de decisión individual la calidad sónica del ambiente tiene un papel moderado, cuando las problemáticas actuales de la ciudad se enfrentan, la mejora acústica ambiental queda en la cola.

Tabla 5 Prioridad declarada de actuación municipal (1=más prioritario)

\begin{tabular}{lccccc}
\hline & \multicolumn{3}{c}{ Le molesta el ruido } & \multicolumn{2}{c}{ Test ANOVA } \\
\hline & $\begin{array}{c}\text { Ranquin } \\
\text { general }\end{array}$ & No & SI & F & Sig. \\
\hline Mejora política de vivienda, p.e: más VPO y alquiler & 1 & 2,5 & 2,4 & 1,32 & 0,27 \\
\hline $\begin{array}{l}\text { Apertura de nuevos espacios verdes, como parques } \\
\text { y jardines }\end{array}$ & 3 & 3,0 & 2,9 & 0,86 & 0,46 \\
\hline $\begin{array}{l}\text { Construcción de más equipamientos, como } \\
\text { bibliotecas y centros de acogida }\end{array}$ & 5 & 3,2 & 3,5 & 1,83 & 0,14 \\
\hline $\begin{array}{l}\text { Mejora del transporte público, como el incremento } \\
\text { de la frecuencia de paso }\end{array}$ & 2 & 2,8 & 2,9 & 0,44 & 0,73 \\
\hline $\begin{array}{l}\text { Reducción del ruido ambiental, como la instalación } \\
\text { de elementos sonoabsorbentes }\end{array}$ & 4 & 3,5 & 3,2 & 1,00 & 0,39 \\
\hline
\end{tabular}

Fuente: elaboración propia 


\section{4.- Resultados de la DAP.}

De las 405 encuestas válidas 110 son de protesta, es decir, el 27\% de las personas encuestadas no manifestó su disposición a pagar como una medida de rechazo al ejercicio. Esta tasa de protesta está ligeramente por encima de lo que es normal en esta clase de valoraciones (Riera, 1994). Lo cual se debe al hecho que los encuestados que protestaron piensan, legítimamente, que no han de pagar por una externalidad generada por otros (aunque ellos/ellas puedan ser parte también del problema); y en todo caso, piensan que el problema tendría que solucionarse con los tributos generales pagados. Lo significativo es que a pesar de este razonamiento general el 73\% de los respondedores haya manifestado su valoración por el silencio, lo cual no hace sino refrendar la magnitud del problema que representa esta externalidad urbana. La DAP promedio del resto de encuestados (ceros verdaderos incluidos) es de 3,39 euros por persona y mes, con una desviación estándar de 3,39 euros. De hecho un $30 \%$ estaba dispuesto a pagar más de 5 euros/persona/mes.

\section{Figura 1 Nivel de hogares con ruidos en el exterior de su vivienda (izq.) y georeferenciación de las encuestas.}

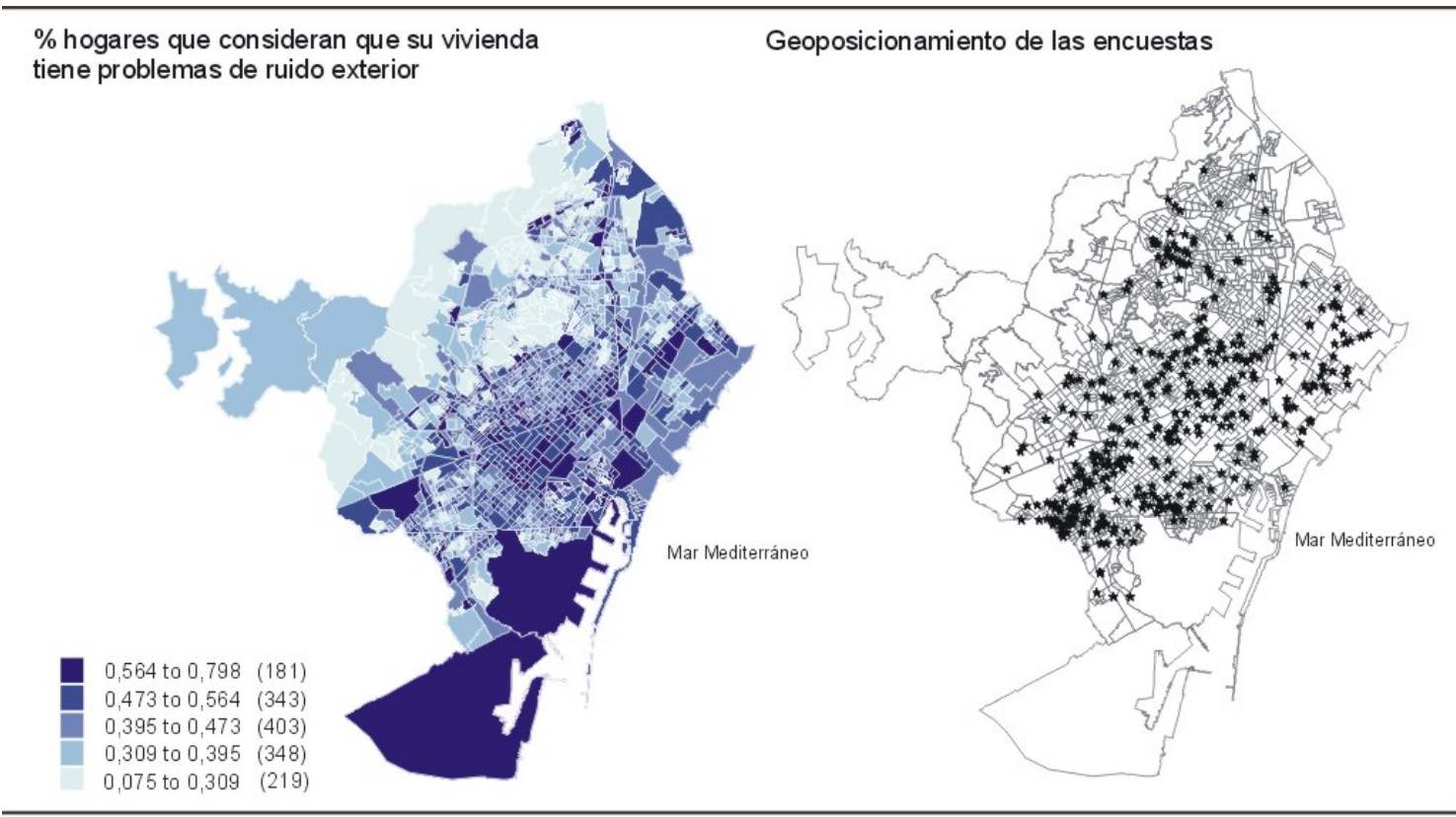

Fuente: Elaboración propia sobre la base del Censo de Población y Vivienda (INE, 2001) y georeferenciación propia

Para explicar la disposición a pagar se ha realizado un análisis de regresión múltiple. A priori se esperaría que la DAP fuese una función del nivel de renta, del perjuicio producido por el ruido, de la consideración del ruido como un agente patógeno, de la edad, del nivel de formación. Toda esta información, como se ha explicado antes, fue recabada en la propia encuesta. Sin embargo, afectos de complementarla se decidió extraer más información contextual del Censo de Población, Vivienda y Locales del Instituto Nacional de Estadística del año 2001. Por esta razón las encuestas fueron geoposicionadas en un sistema de información geográfica, al cual se le añadió una capa de información con datos relativos a: la ocupación socioprofesional de los residentes, el nivel de estudios terminados, el número de hogares que consideran que su vivienda tiene problemas de ruido exterior, el número de locales de diversas actividades 
económicas, y el estado de conservación de las viviendas. A través de una consulta geoespacial los datos del Censo, georeferenciados a escala de sección censal, fueron transferidos a un buffer de 300 metros de radio dibujado a partir del domicilio aproximado de cada persona encuestada. La figura 1 anterior detalla este proceso, en el mapa izquierdo se observa el porcentaje de hogares, por sección censal, que declaró en el Censo tener problemas de ruido en su entorno residencial, mientras que el otro mapa detalla la posición de cada encuesta.

La tabla 6 sistematiza los estadísticos descriptivos de la información utilizada para explicar inferencialmente la DAP de las personas encuestadas. Diferentes variables de control fueron utilizadas, como el nivel de aislamiento en tanto todavía un $25 \%$ de los encuestados vive en casas con carpintería de madera, y solo un $24 \%$ ha realizado obras para protegerse del ruido. Asimismo, se incluyeron variables de percepción como el nivel de molestia (tanto del encuestado como de los hogares del entorno), la percepción del ruido en relación con la salud, la importancia del ruido como factor locativo a la hora de elegir el sitio de residencia, y su importancia en relación con otros problemas ciudadanos. El nivel de exposición potencial al ruido exterior también fue controlado, porque un $57 \%$ de los encuestados tienen viviendas que dan a la vía pública, y un $75 \%$ de éstas son calles, mientras que un $49 \%$ tiene su dormitorio hacia la vía pública, el nivel de la vivienda en relación a la calle también fue controlado. Para analizar la influencia del nivel de renta se utilizó como proxy la ocupación socioprofesional del encuestado, y del entorno en el cual vive (con datos del Censo), en tanto existe una estratificación social marcada en el cual el nivel de renta está asociado a la posición que las personas tienen dentro de la jerarquía laboral. 
Tabla 6 Estadísticos descriptivos de la información utilizada en la construcción del modelo econométrico

\begin{tabular}{|c|c|c|c|c|c|c|}
\hline & & $\mathrm{N}$ & Min & Mean & & Std. Deviation \\
\hline \multirow[t]{53}{*}{$\mathrm{E}$} & El ruido perjudica la salud (1=si) & 405 & 0 & 1 & $90 \%$ & 0,3 \\
\hline & Nivel molestia ruido ( $3=$ mucha) & 405 & 0 & 3 & 1,3 & 1,1 \\
\hline & Carpintería aluminio (1=si) & 405 & 0 & 1 & $56 \%$ & 0,5 \\
\hline & Carpintería plástico (1=si) & 405 & 0 & 1] & $18 \%$ & 0,4 \\
\hline & Carpintería madera (1=si) & 405 & 0 & 1 & $25 \%$ & 0,4 \\
\hline & vidrio doble (1=si) & 404 & 0 & 1 & $50 \%$ & 0,5 \\
\hline & Ha realizado obras contra ruido (1=si) & 405 & 0 & 1 & $24 \%$ & 0,4 \\
\hline & Nivel importancia ruido elec res (4=mucha) & 405 & 0 & 4 & 2,4 & 1,1 \\
\hline & Niv imp próx metro (4=mucha) & 404 & 0 & 4 & 3,2 & 1,0 \\
\hline & Niv imp com vial (4=mucha) & 405 & 0 & 4 & 3,2 & 0,9 \\
\hline & Niv imp equipamientos (4=mucha) & 405 & 0 & 4 & 3,1 & 0,9 \\
\hline & Niv imp calidad y conser urbani (4=mucha) & 405 & 0 & 4 & 3,1 & 0,9 \\
\hline & Niv imp comercio conveniencia (4=mucha) & 405 & 0 & 4 & 2,9 & 0,8 \\
\hline & Niv imp presencia de rest, terraza, bar (4=mucha) & 405 & 0 & 4! & 2,0 & 1,1 \\
\hline & Niv imp seguridad pub (4=mucha) & 405 & 0 & 4 & 3,5 & 0,7 \\
\hline & Niv imp prestigio social (4=mucha) & 405 & 0 & 4 & 2,2 & 1,1 \\
\hline & Niv imp ausencia ruido (4=mucha) & 405 & 0 & 4 & 3,1 & 0,9 \\
\hline & Niv imp prox serv sanit (4=mucha) & 405 & 0 & 4 & 3,0 & 1,0 \\
\hline & Niv imp ausencia industria (4=mucha) & 405 & 0 & 4 & 3,3 & 0,9 \\
\hline & Niv prioridad vivienda (1=más prioritario) & 404 & 1 & 5 & 2,5 & 1,6 \\
\hline & Niv prioridad área verde (1=más prioritario) & 404 & 1 & 5 & 2,9 & 1,2 \\
\hline & Niv prioridad equipamiento (1=más prioritario) & 404 & 1 & 15 & 3,4 & 1,4 \\
\hline & Niv prioridad transporte público (1=más prioritario) & 403 & 1 & 5 & 2,9 & 1,3 \\
\hline & Niv prioridad reducción ruido (1=más prioritario) & 404 & 0 & 5 & 3,3 & 1,4 \\
\hline & Vive junto a calle (1=si) & 402 & 0 & 1 & $75 \%$ & 0,4 \\
\hline & Vice junto a avenida (1=si) & 402 & 0 & 1 & $15 \%$ & 0,4 \\
\hline & vive junto a ronda (1=si) & 402 & 0 & 1 & $2 \%$ & 0,1 \\
\hline & vive junto a peatonal $(1=s i)$ & 402 & 0 & 1] & $3 \%$ & 0,2 \\
\hline & vive junto a plaza (1=si) & 402 & 0 & 1 [ & $4 \%$ & 0,2 \\
\hline & Nivel vivienda & 387 & 0 & 12 & 3,7 & 1,9 \\
\hline & viv da a via pública (1=si) & 401 & 0 & 1 & $57 \%$ & 0,5 \\
\hline & viv da a patio de manzana (1=si) & 401 & 0 & 1 & $16 \%$ & 0,4 \\
\hline & viv da a via y patio de manzana (1=si) & 401 & 0 & 1 & $27 \%$ & 0,4 \\
\hline & dormitorio da a via pública (1=si) & 384 & 0 & 1 & $49 \%$ & 0,5 \\
\hline & dormitorio da a patio de manzana ( $1=s i$ & 384 & 0 & 1 & $45 \%$ & 0,5 \\
\hline & dormitorio da a via y patio de manzana ( $1=s i)$ & 384 & 0 & 1] & $5 \%$ & 0,2 \\
\hline & Propietario (1=si) & 402 & 0 & 3 & $59 \%$ & 0,5 \\
\hline & casado o pareja de hecho (1=si) & 403 & 0 & 1 & $66 \%$ & 0,5 \\
\hline & soltero (1=si) & 403 & 0 & 1 & $21 \%$ & 0,4 \\
\hline & divorciado (1=si) & 403 & 0 & 1 & $8 \%$ & 0,3 \\
\hline & viudo (1=si) & 403 & 0 & 1] & $5 \%$ & 0,2 \\
\hline & tiene hijos (1=si) & 401 & 0 & 1 & $53 \%$ & 0,5 \\
\hline & Estudiante (1=si) & 400 & 0 & 1 & $10 \%$ & 0,3 \\
\hline & Ocupado (1=si) & 400 & 0 & 1 & $71 \%$ & 0,5 \\
\hline & Parado (1=si) & 400 & 0 & 1] & $3 \%$ & 0,2 \\
\hline & Amo de hogar (1=si) & 400 & 0 & 1] & $5 \%$ & 0,2 \\
\hline & Pensionado (1=si) & 400 & 0 & $1 \square$ & $11 \%$ & 0,3 \\
\hline & Nivel de estudios (3=licenciatura) & 405 & 0 & 3 & 1,8 & 0,8 \\
\hline & Hombre (1) & 403 & 0 & 2 & $46 \%$ & 0,5 \\
\hline & Edad & 400 & 13 & 89 & 42,8 & 15,3 \\
\hline & Escala_profesional ( 7 =directiva) & 358 & 0 & 7 & 4,2 & 1,7 \\
\hline & Cualificado (profesional o directivo) & 405 & 0 & 1 & $21 \%$ & 0,4 \\
\hline & Niños $<15$ años viviendo en el hogar & 173 & 0 & 3 & 0,85 & 0,8 \\
\hline \multirow{18}{*}{ c } & Locales de act económica/1.000 personas & 396 & 0 & 802 & 100,29198 & 106,0 \\
\hline & \% directivos en el entorno & 395 & $3 \%$ & $27 \%$ & $11 \%$ & 0,0 \\
\hline & $\%$ profesionales en el entorno & 395 & $5 \%$ & $38 \%$ & $22 \%$ & 0,1 \\
\hline & \% técnicos en el entrono & 395 & $9 \%$ & $20 \%$ & $17 \%$ & 0,0 \\
\hline & \% administrativos en el entorno & 395 & $7 \%$ & $17 \%$ & $13 \%$ & 0,0 \\
\hline & \% dependientes en el entorno & 395 & $6 \%$ & $26 \%$ & $14 \%$ & 0,0 \\
\hline & \% operarios en el entorno & 395 & $2 \%$ & $15 \%$ & $6 \%$ & 0,0 \\
\hline & $\%$ no cualificados en el entorno & 395 & $3 \%$ & $22 \%$ & $8 \%$ & 0,0 \\
\hline & $\%$ analfabetas en el entorno & 395 & $5 \%$ & $12 \%$ & $6 \%$ & 0,0 \\
\hline & $\%$ sin estudios en el entorno & 395 & $5 \%$ & $22 \%$ & $11 \%$ & 0,0 \\
\hline & $\%$ personas con primaria en el entorno & 395 & $10 \%$ & $32 \%$ & $20 \%$ & 0,0 \\
\hline & $\%$ personas con secundaria en el entorno & 395 & $31 \%$ & $48 \%$ & $42 \%$ & 0,0 \\
\hline & $\%$ personas con est universitarios o FP en el entorno & 395 & $4 \%$ & $42 \%$ & $21 \%$ & 0,1 \\
\hline & $\%$ hogares con problemas de ruido exterior & 395 & $21 \%$ & $62 \%$ & $45 \%$ & 0,1 \\
\hline & $\%$ viviendas en el entorno en estado ruinoso & 395 & $0 \%$ & $21 \%$ & $2 \%$ & 0,0 \\
\hline & \% viviendas en el entorno en estado malo & 395 & $0 \%$ & $44 \%$ & $4 \%$ & 0,1 \\
\hline & $\%$ viviendas en el entorno en estado deficiente & 395 & $0 \%$ & $45 \%$ & $16 \%$ & 0,1 \\
\hline & $\%$ viviendas en el entorno en buen estado & 395 & $19 \%$ & $100 \%$ & $78 \%$ & 0,1 \\
\hline
\end{tabular}

Elaboración propia sobre la base de la encuesta ( E)y el Censo de Población, Vivienda y Locales ( C) (INE 2001) 
Para analizar la influencia del nivel de formación se utilizó el nivel de estudios tanto del/a encuestado/a como del entorno. Otras características sociodemográficas como el tipo de hogar, el sexo, la edad y la presencia o no de infantes en el hogar también fueron considerados.

Con el objetivo de medir la elasticidad de la DAP ante la variación de las variables explicativas se utilizó el siguiente modelo:

$$
\operatorname{LnDAP}=C+\sum_{i}^{n} B_{i} X_{i}
$$

En (2) LnDAP es el logaritmo natural de la disposición a pagar, en euros por mes y persona, y $X$ es cada una de las $i$ covariables y factores descritos en la tabla 6 .

Tabla 7 Modelo DAP

\begin{tabular}{|c|c|c|c|c|c|c|c|}
\hline \multicolumn{8}{|c|}{ Model Summary } \\
\hline Model & $\mathrm{R}$ & \multicolumn{2}{|r|}{ R Square } & $\begin{array}{l}\text { Adjusted R } \\
\text { Square }\end{array}$ & $\begin{array}{l}\text { Std. Error of the } \\
\text { Estimate }\end{array}$ & & \\
\hline 7 & ,663(g) & & 0,439 & 0,43 & 0,65 & & \\
\hline $\mathrm{h}$ & \multicolumn{7}{|c|}{$\begin{array}{l}\text { Predictors: (Constant), Nivel molestia ruido, El ruido perjudica la salud, Niv imp ausencia ruido, } \\
\text { Cualificado (profesional o directivo), Carpintería aluminio, Mayores_64, Niv imp ausencia industria }\end{array}$} \\
\hline \multicolumn{8}{|c|}{ ANOVA } \\
\hline Model & & & Sum of Squares & $\mathrm{df}$ & Mean Square & $\mathrm{F}$ & Sig. \\
\hline & 7 Regression & & 90,86 & 7 & 12,98 & 31,11 & 0,000 \\
\hline & Residual & & 115,98 & 278 & 0,42 & & \\
\hline & Total & & 206,84 & 285 & & & \\
\hline \multicolumn{8}{|c|}{ Coefficients } \\
\hline \multirow[t]{2}{*}{ Model } & & & $\begin{array}{l}\text { Unstandardized } \\
\text { Coefficients }\end{array}$ & & $\begin{array}{c}\text { Standardized } \\
\text { Coefficients }\end{array}$ & $\mathrm{t}$ & Sig. \\
\hline & & & $\mathrm{B}$ & Std. Error & Beta & $\mathrm{B}$ & Std. Error \\
\hline \multicolumn{2}{|r|}{7 (Constant) } & - & 0,42 & 0,18 & & 2,31 & 0,022 \\
\hline \multicolumn{2}{|r|}{ Nivel molestia ruido } & & 0,32 & 0,04 & 0,44 & 9,01 & 0,000 \\
\hline \multicolumn{2}{|r|}{ El ruido perjudica la salud } & & 0,42 & 0,12 & 0,17 & 3,40 & 0,001 \\
\hline \multicolumn{2}{|r|}{ Niv imp ausencia ruido } & & 0,13 & 0,05 & 0,13 & 2,53 & 0,012 \\
\hline \multicolumn{8}{|c|}{ Cualificado (profesional o } \\
\hline & Carpintería de aluminio & & 0,22 & 0,08 & 0,13 & 2,81 & 0,005 \\
\hline & Mayores_64 & - & 0,29 & 0,13 & 0,10 & 2,21 & 0,028 \\
\hline & Niv imp ausencia industria & & 0,09 & 0,04 & 0,10 & 2,00 & 0,047 \\
\hline
\end{tabular}

Proceso propio en SPSS 15,0 Método pasos sucesivos

El mejor de los modelos, resumido en la tabla 7 , es capaz de explicar el $43 \%$ de la DAP. El signo de los coeficientes es el esperado y todas las variables mostradas son significativas al $95 \%$ de confianza, mientras que el modelo en conjunto es significativo al $99 \%$ de confianza. El signo de los coeficientes sugieren lo siguiente: cuanto mayor es la molestia ocasionada por el ruido mayor es la DAP, si los respondedores piensan que el ruido perjudica la salud la DAP incrementa, cuanto mayor es la importancia del silencio a la hora de elegir la localización residencial mayor es la DAP, el nivel de renta medida como el hecho o no de que los respondedores fuesen profesionales cualificados incrementa la DAP, mientras que si son 
mayores de 64 años la DAP disminuye, finalmente el nivel de importancia que tiene la ausencia de industria en el entorno residencial incrementa la DAP.

El coeficiente $B$ de la variable nivel de molestia del ruido indica que la elasticidad de la DAP ante la variación del perjuicio es del $32 \%$, es decir, por cada escalón que incrementa el nivel de ruido percibido dentro de la escala ordinal propuesta en la encuesta (por ejemplo, por pasar de una afección media a otra alta), la DAP incrementa en la proporción antes indicada. Los coeficientes beta construidos con las variables tipificadas detallan que el nivel real de afección sonora y la percepción del ruido como un factor maligno para la salud son la principal explicación de la disposición de las personas a pagar por una eventual reducción del ruido ambiental.

Según el modelo las personas de 65 años están menos dispuestas a pagar. La correlación lineal de Pearson entre la edad y el nivel de molestia del ruido es negativa debido a que la presbiacucia produce una pérdida gradual de la audición a medida que envejecemos, especialmente en lo que se refiere a los tonos altos. Según el National Institute of Deafness and Other Communication Disorders entre el 30 y el 35 por ciento de las personas entre 65 y 75 años tiene una pérdida de audición, mientras que este porcentaje se eleva hasta el 50 por ciento para los mayores de 75 años. Por tanto, el hecho que en el modelo de regresión múltiple, que teóricamente controla el efecto del nivel de molestia, la edad avanzada continúe teniendo un signo negativo, es representativo del menor nivel de renta que se percibe tras la jubilación. En consecuencia el efecto parcial de este factor debe entenderse como un indicador de renta estructural.

En cuanto a la carpintería de aluminio se sugiere una causalidad bidimensional, por una parte es de esperar que los hogares más sensibles al ruido tiendan a reformar su vivienda aislándola a través de la sustitución de las ventanas de madera por otras más eficientes en términos acústicos y térmicos, pero también este factor puede estar asociado al nivel de renta, en tanto las viviendas mejor equipadas son también las más caras. Finalmente resulta relevante el hecho que la preocupación por otras externalidades como la ausencia de actividades industriales esté correlacionada con la DAP por reducir el ruido, con lo cual la consciencia ambiental parece ser un fenómeno multidimensional.

Figura 2 prueba de normalidad de los residuos y homocestaicedad

Dependent Variable: LNDAP

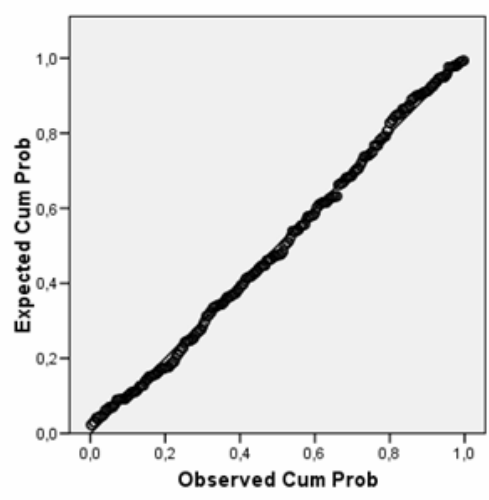

Dependent Variable: DAP euro mes

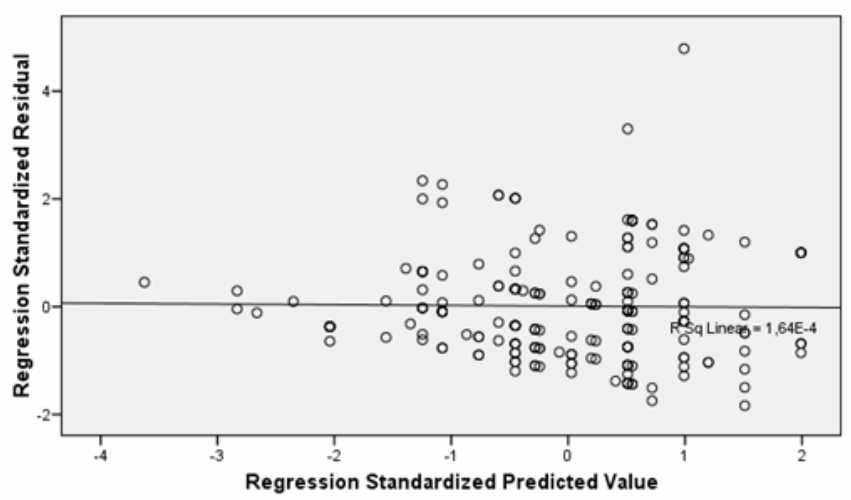

Fuente: elaboración propia 


\section{Conclusiones}

Uno de los principales costes que pagamos cotidianamente por vivir en ciudades densamente urbanizadas es el ruido ambiental. Esta externalidad tiene efectos no sólo en el nivel de confort, que experimentamos cuando nos desplazamos por los espacios públicos como cuando descansamos en nuestros hogares, sino también tiene repercusiones sobre nuestra salud. En definitiva el ruido, es decir, el sonido que por sus características nos resulta molesto, produce una pérdida de bienestar que se traduce en una reducción de calidad de vida. Aunque en ocasiones esta externalidad es en realidad el producto de otros factores que en el fondo son positivos y aumentan las prestaciones urbanísticas de ciertos entornos, como por ejemplo la compacidad y la diversidad de los tejidos urbanos de la ciudad. En este artículo reportamos los resultados de una investigación que tiene por objeto conocer en qué medida la pérdida de bienestar producida por el ruido ambiental se traduce en un valor económico social. Así, el impacto sobre el nivel de bienestar residencial se ha valorado a través de un ejercicio contingente doble que intenta conocer: 1) el nivel de importancia del ruido en relación a otros problemas y atributos urbanos y 2) la disposición a pagar (DAP) por una eventual reducción del ruido. La DAP debe entenderse como un vehículo para valorar el impacto del ruido y no como una propuesta para que las personas afectadas efectivamente contribuyan a mitigar una externalidad negativa originada por terceras personas; aun cuando eventualmente, el ruido urbano es producido de manera colectiva, y en esa medida tendríamos que contribuir en la parte que somos responsables.

La importancia de la ausencia del ruido está en un $6^{\circ}$ lugar dentro de 11 características urbanas propuestas relacionadas con la accesibilidad, la jerarquía social y la calidad ambiental. De hecho el 50,7\% de los 405 respondedores/as declaró que el ruido tenía mucha o bastante importancia a la hora de elegir su localización residencial, mientras que el 50,8\% dijo que el ruido le provocaba una bastante o mucha molestia. Estos resultados dejan ver que desde la perspectiva de la percepción de las personas el ruido ambiental es una externalidad negativa que afecta con cierta severidad a casi la mitad de la población barcelonesa. Sin embargo, cuando se preguntó por el nivel de prioridad que tenía su reducción a través de políticas públicas éste quedó en $4^{\circ}$ lugar dentro de un conjunto de 5 acciones propuestas.

El 73 por ciento de las personas encuestadas revelaron su DAP, siendo ésta de media equivalente a 3,39 euros por persona y día para una reducción sonora equivalente a pasar de un nivel de ruido característico de una hora punta de un día laborable a otro característico del nivel que se experimenta a las 21:00 horas del mismo día. Esta DAP equivale, aproximadamente $^{9}$, a un $0,28 \%$ de la renta bruta familiar disponible del municipio de Barcelona, Barreiro et al. (2005) han reportado para un nivel de reducción sónica similar un porcentaje muy parecido $(0,27 \%)$ para el caso de Pamplona. Para analizar el comportamiento de los participantes su DAP fue analizada mediante un análisis de regresión múltiple. Mediante la ayuda de un sistema de información geográfica, la información individual de cada respondedor/a fue complementada con otros datos provenientes del contexto de la vivienda del/a respondedor/a definido por un buffer de 300 metros de radio. El modelo econométrico capaz de explicar el $43 \%$ de la DAP es coherente con la teoría en tanto, la DAP aumenta a medida que incrementa el nivel de molestia producido por el ruido, cuando se cree que éste

\footnotetext{
${ }^{9}$ La renta bruta familiar disponible de referencia es del año 2002 es decir la última publicada por el Departament d'Estadística de l'Ajuntament de Barcelona.
} 
perjudica la salud, cuando el ruido es un determinante en la elección residencial, así como también lo es la ausencia de industria. En sentido contrario la DAP disminuye en el caso de los respondedor/a de más de 64 años debido a que las rentas del trabajo también disminuyen tras la jubilación, considerando el hecho que la menor perturbación producida por el ruido en las personas mayores ya ha sido controlado por la variable que mide el nivel de molestia que produce el ruido.

Cabe concluir señalando la importancia de avanzar en este tipo de investigaciones que permitan conocer en toda su magnitud el impacto económico social del ruido, por ejemplo el que se produce, más allá de la vivienda, sobre la habitabilidad del espacio público. Esta debería ser la base para la generación de políticas urbanísticas socialmente más rentables; fundadas más que en el control, en la conciencia de preservación colectiva de nuestro entorno ambiental más inmediato: la ciudad.

"Pues ¿qué? ¿No será alguna vez más cómodo estar libre de todo tumulto? [...] ¿Qué necesidad de torturarse más tiempo, si Ulises encontró tan fácilmente para sus compañeros el remedio adecuado contra las sirenas?"

Lucio Anneo Séneca 


\section{Bibliografía.}

BARREIRO, J., M. SANCHEZ, and M. VILADRICH-GRAU. How Much are People Willing to Pay for Silence? A Contingent Valuation Study. Applied Economics. 2005, 37(11), pp 1233-1246.

BECKER, N. \& LAVEE, D. The benefits and Costs of Noise Reduction. Journal of Environmental Planning and Management, 2003, Vol. 46, Num. 1, pp. 97-111.

BJORNER, T. Combining socio-acoustic and contingent valuation surveys to value noise reduction, Transportation research part d-transport and environment 9 (5): 341-356 SEP 2004

BROOKSHIRE, D. S., et al. Valuing Public Goods: A Comparison of Survey and Hedonic Approaches. The American Economic Review. 1982, 72(1), pp 165-177.

DAUMAL, F. Arquitectura Acústica: poética y diseño. Edicions UPC, Barcelona, 2001.

DAUMAL, F. y DE GORTARI, J. La influencia del ruido en el precio de la vivienda, Congreso Nacional de Acústica (Tecnicústica 2006-37), Encuentro Ibérico de Acústica, EAA European Symposium on Hidroacustics, Gandia, Octubre 2006.

GARCÍA, B. \& GARRIDO, F. La contaminación acústica de nuestras ciudades, Colección Estudios Sociales, Núm. 12. Fundación La Caixa, 2003, Barcelona.

MARMOLEJO,C. La incidencia de la percepción del ruido ambiental sobre la formación espacial de los valores residenciales: un análisis para Barcelona, forthcoming, Revista de la Construcción, 2008.

MARMOLEJO,C. \& ROMANO, J. La valoración económica social del ruido aeroportuario. Un análisis para el entorno residencial del Aeropuerto de Barcelona, Proceedings de la 15th Annual European Real Estate Society Conference, Krakow, 2008.

NIDCD, National Institute of Deafness and Other Communication, Disponible en línea http://www.nidcd.nih.gov/health/spanish/presbycusis span.asp [Consulta: 20/4/08]

NAVRUD, S. Economic benefits of a program to reduce transportation and community noise-A contingent valuation survey. In: Proceedings of Internoise 2000, 200, Nice.

RIERA, P. Manual de Valoración Contingente, Instituto de Estudios Fiscales, 1994, Madrid. Disponible en línea: http://selene.uab.es/prieram/papers.htm [Consulta: 18/3/08]

RIERA, O. Fundamentos Teóricos de la Valoración Contingente, apuntes de clase Disponible en línea: http://selene.uab.es/prieram/valuation/cvevgraf.pdf, 2005 [Consulta: 25/4/08] 
ROSEN, S. Hedonic prices and implicit markets: production differentiation in pure competition. Journal of Political Economy, 1974, Num. 82, pp. 34-55.

SAELENSMINDE, K. Stated choice valuation of urban traffic air pollution and noise, Transportation Research Part D-Transport and Environment. 1999.

SCARPA, R. \& WILLIS KG. Distribution of willingness-to-pay for speed reduction with nonpositive bidders: Is choice modeling consistent with contingent valuation? Transport Reviews, Vol. 26, Issue 4, 451-169, July 2006.

VAINIO, M. Comparison of hedonic prices and contingent valuation methods in urban traffic noise context, in Proceeding of Internoise 2001 (Ed.) R. Boone Acoustical Society of The Netherlands. 2001 The Hage.

WALTERS, A. A. Noise and Prices. Oxford University Press, London, 1975. 